\title{
Emergent Learning Theory of Putting Exercise Blending into Learning
}

\author{
Gengdan $\mathrm{Hu}$ \\ Department of Psychology \\ Tongji University \\ Shanghai, China 200092
}

\begin{abstract}
In view of the negative impact of the Internet on students' academic performance and physique health, this article points out the misunderstanding of the academic circles and relevant government departments' policies on solving these two issues above. It insists that the effective way to solve these two issues at present is to find a new learning theory paradigm that can not only help students grasp knowledge quickly, improve their academic performance, but also maintain and improve their physical and mental health. This article puts forward a learning method that is not only conducive to the development of students' cognitive ability, but also contributes to their physical and mental health, i.e. emergent learning theory of putting exercise blending into learning (referred to as emergent learning theory below), and makes a preliminary discussion on the effect of emergent learning theory and its mechanism through empirical research.
\end{abstract}

Keywords-emergent learning theory; physical exercise; physique; Internet addiction (IAD); cognitive ability

\section{INTRODUCTION}

Under the exam-oriented education and the command of the college entrance examination, the primary and secondary school students chronically face a very heavy learning task. Even after entering the university, they have to continue to bear great learning pressure in the face of increasingly severe employment pressure and competition for postgraduate entrance examination, which leads to a series of problems such as the decline of teenagers' physique, serious psychological issues and the increase of suicide rate (Ma, 2012). In response to this phenomenon, many scholars have proposed reform measures and recommendations such as burden reduction, accountability, adding sports to college entrance examination, and the cancellation of the college entrance examination (Wen \& Hu, 2007; Shen \& Wu, 2012), but they have little effect because these measures and recommendations are either divorced from reality or lack of operability (Hu \& Xu, 2018). Therefore, the author believes that the only way to solve this problem at this stage is to find a new learning theory that can not only help students quickly grasp knowledge, improve their academic performance, but also maintain and improve their physical and mental health. Through many years of teaching practice and personal experience, the author preliminarily has summarized a learning method that is conducive to the development of students' cognitive ability and physical and mental health, i.e. emergent learning theory. This study attempts to make a preliminary discussion of this learning theory through empirical research.

\section{The CONNOTATION, STEPS AND MECHANISM OF EMERGENT LEARNING THEORY}

\section{A. The Connotation and Steps of Emergent Learning Theory}

Firstly, quickly reviewing the knowledge learned during the day in classroom and read the review materials and exercise books. Secondly, thinking of and understanding knowledge while walking alone, retelling, analyzing and summarizing the contents above. Thirdly, combing and condensing knowledge points, key points and difficult points, transition points. Then, discovering problems and finding solutions. Later, consolidating knowledge and emerging new knowledge structures. Finally, one can achieve his learning goals to maintain physical health, enhance academic performance and innovation ability.

\section{B. The Possible Mechanism of Improving Learning Achievement and Physique of Emergent Learning Theory}

The retelling and independent thinking while walking can play a unique role in the development of students' innovative ability (e.g. memory, imagination, insight, and attention) and logical thinking. In addition, walking itself is a kind of positive rest, which is conducive to relieving brain fatigue, releasing physical and mental stress, as well as enhancing cardio-pulmonary function. When the cardio-pulmonary system works with higher capacity, the brain is at its peak and the ability to think creatively will be improved obviously. Therefore, they promote each other and complement each other to form a beneficial and positive cycle.

\section{METHOD}

\section{A. Subjects}

60 students of high school freshmen with sedentary and little exercise were selected as the subjects (40 males and 20 females) through Middle School Students' Daily Behavior Habits Questionnaire, and they were randomly divided into 
experimental group and control group. Each group consists of 30 people ( 20 males and 10 females).

\section{B. Tools}

1) Middle school students' daily behavior habits questionnaire: This self-edited questionnaire has a total of 30 questions, including 5 dimensions: moral standard, learning attitude, physical exercise, work and rest, and Internet access, each dimension has 6 questions, using the expert consultation method to test and modify the structural validity of the questionnaire.

2) Symptom self-rating checklist (SCL-90): SCL-90 has a total of 90 projects, from feeling, thinking, consciousness, behavior to living habits, interpersonal relationships, eating and sleeping aspects, etc. to assess whether a person has some kind of psychological symptoms and its severity. The nine factors tested are somatization, obsessive-compulsive symptoms, interpersonal sensitivity, depression, anxiety, hostility, terror, paranoia, and mental illness. Diet, sleep, etc. are not included in the nine factors. The average of each factor is usually used as the evaluation index (Wang, 1984).

3) Questionnaire on IAD of middle school students: Chinese Internet Addiction Scale (CIAS) (Chen, Weng, \& $\mathrm{Su}$, et al., 2003), is composed of 26 questions, contains 5 factors: tolerance, withdrawal response, compulsive Internet use, time management issues, and interpersonal health issues. CIAS is marked according to Richter 4 point score, the total score of which represents degree of personal IAD, the higher the score is, the higher IAD tendency is. The specific evaluation criteria: 42 points below as general web surfers, $42-59$ points as mild addicts, 59 points above as severe addicts.

4) Physique testing instruments: height and weight tester, lung capacity tester, electronic grip tester, type sitting body Front flexor tester, sports stopwatch, ruler, gymnastics pad, horizontal bar.

\section{Experiment Comparison}

For the subjects in the experimental group who adopted emergent learning theory, they conducted the learning intervention alone after dinner or evening, 5 times a week (from Monday to Friday), 45-60min each time, with a walking distance of about $2-3 \mathrm{~km}$ every time and a speed of $90-100 \mathrm{~m}$ per minute. This is because fast pace will affect the allocation of attention resources, leading to the reduction of subjects' attention to the problem, which affects their memory processing, thus affecting the effect of intervention. The less attention the brain spends on completing a walking action, the better the intervention effect is. As a result, walking required by this experiment completes unconsciously. Walking places need to meet three conditions: safe, familiar, relatively quiet and less photoelectric stimulation. In case of bad weather (such as wind and rain, fog and haze), walking can be changed to indoor stadium, wind and rain corridor or similar other places.

The control group followed their usual learning habits and methods. The teaching content, teaching requirements, teaching progress, test and examination, daily school sports activities and other aspects of the two groups were exactly same. In addition, with the cooperation of parents, the two groups of subjects were required to maintain consistency in diet, rest, sleep and other lifestyles as far as possible, to keep the conditions of the two groups consistent to the maximum.

Experimental time: In the first semester of senior one, the experimental period was 4 months. Before the experiment (the first week of teaching) and after the experiment (the 16th week of teaching), the daily behavior norms, physical condition, IAD status and mental health level of the two groups were tested respectively. The results of their senior high school entrance examinations (Chinese, mathematics and English) were used as the scores before the experiment, and the results of the final examinations (Chinese, mathematics and English) were taken as the scores after the experiment. Finally, the intervention effect of emergent learning theory was evaluated by the pair-to-pair comparison of the daily behavior norms, academic performance, physique test results, IAD status and mental health level of the two groups before and after the experiment.

\section{Statistical Approach}

The following data were analyzed with SPSS22.0, and the comparison between the groups was tested by pairedsamples $\mathrm{t}$ test, $\mathrm{p}<0.05$ was considered as statistically significant difference.

\section{RESULTS}

\section{A. The Performance of the Two Groups}

Before the experiment, there was no significant difference between the two groups regarding Chinese, mathematics and English achievements in senior high school entrance exams, $p>0.05$. After the experiment, there was a very significant difference in mathematics performance between the two groups, $\mathrm{p}<0.01$; Performances of Chinese and English in the experimental group were respectively better than the control group's, $\mathrm{p}<0.05$. (Table I).

TABle I. The Comparison of ACADEmic Performances of Two Groups Before AND After the EXPeriment $(\mathrm{X} \pm \mathrm{SD})$

\begin{tabular}{|l|l|l|l|l|}
\hline \multirow{2}{*}{ Courses } & Before & After \\
\cline { 2 - 5 } & Experimental group (30) & Control group (30) & Experimental group (30) & Control group (30) \\
\hline mathematics & $90.47 \pm 22.30$ & $91.12 \pm 24.23$ & $102.57 \pm 19.41^{* *}$ & $90.34 \pm 22.95$ \\
\hline Chinese & $94.66 \pm 19.83$ & $93.95 \pm 19.20$ & $101.84 \pm 17.94^{*}$ & $94.46 \pm 18.98$ \\
\hline English & $94.56 \pm 21.37$ & $95.19 \pm 19.77$ & $103.78 \pm 18.45^{*}$ & $95.62 \pm 19.23$ \\
\hline
\end{tabular}




\section{B. Two Groups of IAD and Psychological Status}

"Table II" shows that there was no significant difference in IAD time and weekly online time between the two groups before the experiment, $\mathrm{p}>0.05$; compared with the control group, the IAD score and online time per week of the experimental group were significantly reduced after the experiment, $\mathrm{p}<0.01$. As can be seen from "Table III", in addition to paranoia, somatization, terror and psychotic scores, other factors such as obsessive-compulsive disorder, hostility, interpersonal relationship, depression, and anxiety scores decreased significantly after intervention, the differences between the two groups were significant, or more significant, $\mathrm{p}<0.05$ or $\mathrm{p}<0.01$.

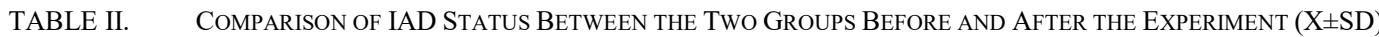

\begin{tabular}{|l|l|l|l|l|}
\hline \multirow{2}{*}{ Testing index } & Before & After \\
\cline { 2 - 5 } & Experimental group (30) & Control group (30) & Experimental group (30) & Control group (30) \\
\hline IAD score & $58.39 \pm 10.83$ & $57.54 \pm 8.33$ & $42.4 \pm 8.85^{* *}$ & $55.3 \pm 11.31$ \\
\hline Internet time per week & $10.41 \pm 3.27$ & $9.83 \pm 3.85$ & $6.45 \pm 1.82^{* *}$ & $10.36 \pm 2.62$ \\
\hline
\end{tabular}

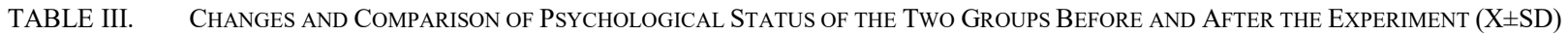

\begin{tabular}{|c|c|c|c|c|}
\hline \multirow[b]{2}{*}{ Psychological indicator } & \multicolumn{2}{|l|}{ Before } & \multicolumn{2}{|l|}{ After } \\
\hline & Experimental group (30) & Control group (30) & Experimental group (30) & Control group (30) \\
\hline Obsessive-compulsive disorder & $1.98 \pm 0.60$ & $1.88 \pm 0.63$ & $1.67 \pm 0.61 *$ & $1.94 \pm 0.65$ \\
\hline Paranoia & $1.80 \pm 0.71$ & $1.83 \pm 0.65$ & $1.60 \pm 0.68$ & $1.82 \pm 0.63$ \\
\hline Hostile & $1.72 \pm 0.68$ & $1.74 \pm 0.73$ & $1.41 \pm 0.70 * *$ & $1.76 \pm 0.72$ \\
\hline Interpersonal relationship & $1.88 \pm 0.68$ & $1.89 \pm 0.71$ & $1.53 \pm 0.69 * *$ & $1.93 \pm 0.70$ \\
\hline Depression & $1.74 \pm 0.67$ & $1.78 \pm 0.63$ & $1.40 \pm 0.68 * *$ & $1.82 \pm 0.74$ \\
\hline Anxiety & $1.61 \pm 0.58$ & $1.74 \pm 0.65$ & $1.43 \pm 0.63 *$ & $1.80 \pm 0.61$ \\
\hline Somatization & $1.48 \pm 0.55$ & $1.51 \pm 0.49$ & $1.49 \pm 0.49$ & $1.59 \pm 0.42$ \\
\hline Terror & $1.43 \pm 0.52$ & $1.52 \pm 0.58$ & $1.41 \pm 0.49$ & $1.49 \pm 0.52$ \\
\hline Psychotic & $1.60 \pm 0.67$ & $1.64 \pm 0.65$ & $1.52 \pm 0.64$ & $1.67 \pm 0.68$ \\
\hline
\end{tabular}

\section{Physique Condition of the Two Groups}

"Table IV" and "Table V" show that there was no significant difference in physique test scores between the two groups before the experiment. But, after the experiment, the experimental group's grip strength index and long run (1000m running or $800 \mathrm{~m}$ running) scores were significantly better than the control group's, and the differences between the two groups were significant, $\mathrm{p}<0.05$; the vital capacity and body mass index was very significant, $\mathrm{p}<0.01$; the experimental group's body mass index (BMI) and sitting body flexion scores were better than the control group's, but the differences between the two groups were not significant, and other indicators were not significantly different.

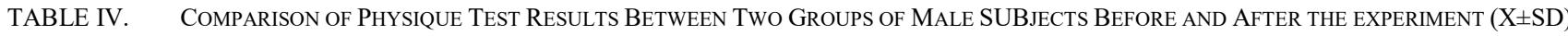

\begin{tabular}{|c|c|c|c|c|}
\hline \multirow[t]{2}{*}{ Test index } & \multicolumn{2}{|l|}{ Before } & \multicolumn{2}{|l|}{ After } \\
\hline & Experimental group (30) & Control group (30) & Experimental group (30) & Control group (30) \\
\hline Height $(\mathrm{cm})$ & $170.11 \pm 5.33$ & $170.38 \pm 5.03$ & $171.59 \pm 4.88$ & $171.5 \pm 5.12$ \\
\hline Weight (kg) & $59.78 \pm 8.74$ & $59.37 \pm 8.72$ & $59.43 \pm 8.37$ & $61.72 \pm 7.94$ \\
\hline BMI $\left(\mathrm{kg} / \mathrm{m}^{2}\right)$ & $20.64 \pm 2.79$ & $20.52 \pm 2.93$ & $20.05 \pm 2.78$ & $20.62 \pm 2.84$ \\
\hline $\begin{array}{l}\text { Vital capacity and body mass } \\
\text { index }(\mathrm{ml} / \mathrm{kg})\end{array}$ & $63.08 \pm 11.54$ & $62.51 \pm 10.83$ & $72.36 \pm 10.94 * *$ & $64.45 \pm 11.06$ \\
\hline Grip strength index & $65.72 \pm 10.24$ & $64.97 \pm 11.62$ & $72.56 \pm 9.86^{*}$ & $67.20 \pm 10.51$ \\
\hline Sitting body flexion $(\mathrm{cm})$ & $10.82 \pm 8.05$ & $10.53 \pm 7.95$ & $12.58 \pm 7.40$ & $11.70 \pm 7.86$ \\
\hline Standing long jump $(\mathrm{cm})$ & $233.52 \pm 21.08$ & $229.18 \pm 20.98$ & $236.62 \pm 19.87$ & $232.97 \pm 20.02$ \\
\hline Pull-up & $4.97 \pm 2.94$ & $5.21 \pm 3.87$ & $6.15 \pm 3.54$ & $5.96 \pm 3.05$ \\
\hline $50 \mathrm{~m}$ running $(\mathrm{s})$ & $7.97 \pm 0.51$ & $7.90 \pm 0.61$ & $7.78 \pm 0.58$ & $7.87 \pm 0.63$ \\
\hline $1000 \mathrm{~m}$ running $(\mathrm{s})$ & $259.48 \pm 14.67$ & $261.35 \pm 15.82$ & $250.36 \pm 15.14 *$ & $258.33 \pm 15.62$ \\
\hline
\end{tabular}

TABLE V. COMparison of Physique Test Results Between Two Groups of Female SUBJeCts Before And After the EXPeriment (X \pm SD)

\begin{tabular}{|c|c|c|c|c|}
\hline \multirow[t]{2}{*}{ Test index } & \multicolumn{2}{|l|}{ Before } & \multicolumn{2}{|l|}{ After } \\
\hline & Experimental group (30) & Control group (30) & Experimental group (30) & Control group (30) \\
\hline Height $(\mathrm{cm})$ & $158.51 \pm 5.05$ & $158.06 \pm 5.36$ & $160.13 \pm 5.20$ & $159.82 \pm 5.64$ \\
\hline Weight $(\mathrm{kg})$ & $50.42 \pm 7.33$ & $50.82 \pm 7.50$ & $50.41 \pm 6.86$ & $52.28 \pm 7.13$ \\
\hline BMI $\left(\mathrm{kg} / \mathrm{m}^{2}\right)$ & $20.15 \pm 2.75$ & $20.58 \pm 2.68$ & $19.81 \pm 2.67$ & $21.10 \pm 2.83$ \\
\hline $\begin{array}{l}\text { Vital capacity and body mass } \\
\text { index }(\mathrm{ml} / \mathrm{kg})\end{array}$ & $56.81 \pm 10.33$ & $57.22 \pm 10.74$ & $66.73 \pm 10.12^{* *}$ & $57.89 \pm 10.36$ \\
\hline Grip strength index & $48.42 \pm 8.57$ & $48.96 \pm 8.15$ & $55.15 \pm 8.33^{*}$ & $50.22 \pm 8.86$ \\
\hline Sitting body flexion $(\mathrm{cm})$ & $12.74 \pm 6.15$ & $12.22 \pm 6.62$ & $14.15 \pm 5.93$ & $12.76 \pm 6.07$ \\
\hline Standing long jump (cm) & $176.64 \pm 17.10$ & $176.15 \pm 17.54$ & $180.13 \pm 16.85$ & $178.45 \pm 17.02$ \\
\hline Sit-ups (times/per min) & $36.59 \pm 6.94$ & $36.23 \pm 7.06$ & $38.82 \pm 7.12 *$ & $36.53 \pm 7.30$ \\
\hline $50 \mathrm{~m}$ running $(\mathrm{s})$ & $9.24 \pm 0.60$ & $9.32 \pm 0.58$ & $9.02 \pm 0.53$ & $9.20 \pm 0.60$ \\
\hline 800 running $(\mathrm{s})$ & $257.22 \pm 19.16$ & $257.75 \pm 18.77$ & $244.23 \pm 17.92 *$ & $256.86 \pm 18.35$ \\
\hline
\end{tabular}




\section{DISCUSSION}

\section{A. The Influence of Emergent Learning Theory on Academic Achievements and Its Mechanism}

With regard to the causes of the continuous decline of juvenile physique in China, the mainstream viewpoint of academic circles is that they derive from examinationoriented education and college entrance examination (Zhang, 2014). For example, according to a survey conducted by China Youth News on who should bear the main responsibility for the continuous decline of juvenile physique, $67.9 \%$ of the interviewees preferred the examinationoriented education system as the top reason.

Some researches have shown that students with low learning efficiency can be divided into two types. The first type is those who are extremely hardworking, sitting sill and using all disposable time for learning for their future instead of taking physical exercise, thinking that their diligence would be rewarded. However, the real effect turns out to be mental and physical exhaustion, having declined physique, learning efficiency and academic scores due to the contravention of "living clock". Because this unsustainable and unscientific learning method violates the cognition and operation principle of human brain (Scudder, Federmeier, \& Raine, et al., 2014).

The other is the students with weak self-control and easy distraction. They are vulnerable to external interference such as the Internet and TV. As a result, it seems on the surface that a lot of time is invested in learning, but in fact, most of it is ineffective time, which leads to low learning efficiency and occupies the time that should be used for physical exercise, resulting in long sitting and little movement, psychological imbalance and physical deterioration.

Therefore, it is particularly important to explore a new learning method, which can not only improve the students' performance but also prevent their physique deterioration, while leaving the college entrance system unchanged. The "emergent learning theory" proposed in this article is based on this. The result of the intervention experiment showed that, compared with the control group, students in the experimental group had an obvious improvement in mathematics, Chinese and English scores after the experiment (Table I), which indicates that there is a significant relationship between emergent learning theory and the improvement of subjects' academic performance. The mechanism can be analyzed as follows.

Firstly, timely reviewing daily courses is in accordance with the law of Ebbinghaus's memory curve. Ebbinghaus pointed out that memory can be divided into instantaneous memory, short-term memory and long-term memory, and the forgetting speed turns from fast to slow. The fastest forgetting sections were 42 percent, 56 percent, and 66 percent at 20 minutes, 1 hour, and 24 hours, respectively (Hermann, 1885). It can be seen that according to emergent learning theory, retelling after dinner or in the evening is helpful to memorize the new knowledge learned by day and consolidate the previous content.
Secondly, when walking outside, students cannot touch the written content. If students want to retell, analyze, deduce and summarize the knowledge they have learned in this situation, they must construct the knowledge block diagram in their brains, clarify the concept points and specific application methods. In this process, first of all, the knowledge that students do not fully grasp can be found, which is helpful for them to supplement and consolidate later; Secondly, through individual thinking and imagination, the effect of understanding memory is better than that of memorizing words in front of books (Yang, 2008). Studies have confirmed that the influence of knowledge structure diagram is greater than that of words (Chen \& Yang, 2014). Therefore, the construction of knowledge structure diagram is helpful for students to have a deeper and more comprehensive grasp of knowledge.

Finally, physical exercise is closely related to the improvement of brain flexibility and thinking ability of the brain. Studies have showed that children's nervous system develops much faster than their muscles from primary school to middle school. At this time, if students can participate in sports activities, they can improve the function of the nervous system (Sun, 2000), which is conducive to the improvement of cognitive ability, thus enhancing students' innovation, thinking ability and academic performance. Moderate and low intensity physical exercise can effectively improve the cardiopulmonary function of the subjects. When the heart and lung work at a higher level, the brain can be at its peak. Students with poorer cardiorespiratory function may be more prone to dozing off in class and have lower learning efficiency, and emergent learning theory with walking can just solve this problem.

In summary, emergent learning theory can effectively improve the academic performance of the subjects from the perspective of improving their cognitive abilities such as memory, attention, imagination, insight and logical thinking.

\section{B. The Influence of Emergent Learning Theory on Adolescents' IAD and Psychology and Its Reasons}

As can be seen from "Table II" and "Table III", after the intervention experiment, the score of IAD, the time spent online every week and some psychological indicators in the experimental group all tended to improve significantly. According to the characteristics of the subjects in this study, the author believes that the reasons for this result can be divided into two aspects.

On the one hand, for the students who sit too much and study hard, their academic performance is relatively good. Research has showed that $40 \%$ to $50 \%$ of outstanding students have the characteristics of "psychological syndrome of excellent students" to varying degrees (Qian, 2004). They attach great importance to the success of exams and have great mental burden, and often show symptoms such as excessive tension, anxiety and depression. Besides, they may form a wrong self-conscious and value because they have been always praised by parents and teachers due to great academic achievements, and have weak psychological tolerance in the face of setbacks. Under multiple pressures, 
they are likely to choose the Internet as the outlet for stress relief. And emergent learning theory can solve these problems effectively. The application of this learning theory, one is to make students have a certain amount of time every day in the fresh, open outdoor leisurely walk, helps alleviate the symptoms of depression, anxiety, make people feel comfortable; Both can obviously improve grades, make the student's study pressure decrease relatively, so as to have more time, energy, communication with parents, students and teacher exchanges, the interpersonal relations, learning attitude, life will be accordingly to actively, to get to the Internet as a pressure vent desire will effectively reduce the negative way.

A study showed that physical exercise, especially longterm physical exercise, has good effect on promoting mental health and controlling psychosomatic diseases. The significance of the national fitness program being carried out in China lies not only in physical exercise, but also in mental exercise (Zhang \& Mao, 1995).

It can be seen that sticking to exercise and forming the habit of long-term exercise not only have a positive impact on learning, but also can maintain and improve mental and physical health, which has long-term significance for everyone (Hu, Qian, \& Lin, 2013; Wang \& Tong, 2003). Emergent learning theory is an indispensable part to promote the formation of teenagers' sports habits in this era of Internet.

On the other hand, as for the students with low learning efficiency who are also used to sitting still for a long time, moving as little as possible, and having trouble to concentrate, they always easily daze, gossip, or even begin to surf the Internet with mobile phone unconsciously and uncontrollably when facing dull texts and questions. Gradually they would rely on this way of escaping and become cellphone complexes or phubbers. Prolonging in a low head position is likely to cause students' weakening of the cervical and cervical muscles, increase the burden on the posterior muscles of the neck, make part of the neck and shoulders hard, cause blood circulation reflux obstacles (Ding, Zhang, \& Li, et al., 2015). It also causes psychological unhealthiness. Related researches have shown that more than half of the adolescents feel anxious and unsafe when missing mobile phones, which will lead to a sudden drop of their academic performances (Li \& Yuan, 2013).

The reason of the emergence of phubbers is that many students are tired of endless, ever-increasing learning tasks with increasing difficulty, while mobile-phones provide them with another world to escape from the reality. However, they still have intermittent guilt about avoiding learning. Emergent learning theory can help students prevent cellphone complex from being formed. One of its elements is a solitary, relaxing walk outdoors, it has a positive and effective substitute effect in relieving students' learning pressure. In addition, what they have learned can be effectively consolidated and reviewed in the process of walking and thinking, so that their confidence in the face of new learning tasks can be significantly enhanced. In this study, all the subjects in the experimental group expressed their willingness to accept this learning method, which is not only conducive to reducing learning pressure and improving learning effect, but also able to adjust their psychology and develop the habit of independent exercise.

In conclusion, emergent learning theory can not only effectively alleviate teenagers' depression, anxiety, interpersonal tension and other psychological problems, but also is an effective way to make cellphone complexes return to the real world from the virtual Internet world.

\section{The Influence of Emergent Learning Theory on Adolescents' Physique and Its Mechanism}

As mentioned in the introduction of this paper, many scholars and relevant functional departments advocate that measures such as "burden reduction" and "accountability" should be implemented to reduce students' learning pressure and improve teenagers' physique. At present, the government functional departments of many provinces and cities have introduced relevant measures to put these recommendations into practice. For example, in 2009 Shanghai municipal education commission requested the establishment of a bulletin system on the implementation of curriculum in primary and secondary schools, so as to effectively reduce the heavy workload of primary and secondary students. Since 2013, Shanghai has implemented the "grading system" evaluation based on curriculum standards in the first and second grades of primary schools. There is no written examination, no score evaluation, no ranking, etc. In 2016 Shanghai municipal primary school tried to further "reduce the burden" on the first and second grade students: the number of class hours was reduced, for example, the number of English class hours was reduced from 4 to 3 a week and the school time was advanced from 16:00 PM to 15:20 PM every day. Some regions have introduced "accountability" through aggressive legislation. Kunming, for example, has proposed that if students' physical health status in a school declines for three consecutive years, the head of the education bureau at the county level will be severely held accountable and the principal and direct heads of the school will be given administrative sanctions according to law.

However, the implementation effect is not satisfactory, far from the desired goal.

The National Sleep Foundation of the United States pointed out that 8-hour sleep not only helps to consolidate the procedural and declarative memory of new knowledge, but also reduces the subjective pressure on adolescents (Cedernaes, Rångtell, \& Axelsson, et al., 2015), while 80\% of students in China sleep less than 8 hours every day. In contrast, domestic surveys have shown that few teenagers exercise for one hour every day. Multiple surveys have showed that playing online games and surfing the Internet occupy students' exercise time (Tao \& Li, 2005; Li, 2013). Therefore, it can be inferred that one of the reasons for the continuous physique decline of domestic teenagers is that students always spend the time released by school subtraction on self-pressurized study or surfing the Internet or watching TV programs, instead of physical exercise and sleep. 
The results of physique fitness test in this study showed that (Table IV, Table V) after the intervention experiment, the physique fitness index of the experimental group was better than that of the control group, especially the results of vital-capacity-and-weight-index, grip strength BMI, sit-ups, middle-distance running $(800 \mathrm{~m} / 1000 \mathrm{~m})$ have changed significantly or very significantly. The reasons why emergent learning theory can effectively improve students' physique can be discussed from the following three aspects.

Firstly, walking is a form of aerobic exercise. And the effect of exercise on improving the physique of teenagers has been confirmed by academic circles ( $\mathrm{Hu}, 2018)$. Exercises such as swimming, jogging, cycling, all kinds of sports balls all repeated rhythm motion participated by the motion of the big muscle group, which can effectively accelerate blood circulation, simulate metabolism and the secretion of growth hormone; Physical exercise not only improves bone and muscle strength, but also improves the function of the internal organs and nervous system.

Secondly, walking itself is a positive way of rest, which releases mental tiredness, mental and physical pressure and enhances cardio-pulmonary function. A foreign study has shown that walking can not only make muscles and bones stronger, but also that the women who have been walking on regular basis for 10 to 15 years have a lower risk of heart disease than their less active sisters, which is consistent with the results of physical intervention in this study (Elaine, Linda, \& Mohammed, et al., 2015). It should be pointed out that the exercise prescription for improving physique through walking requires that the amount of exercise should reach 90-120 m/minute, 40-50 min/day, 3-4 times/week. Emergent learning theory can just meet this requirement.

Thirdly, emergent learning theory can effectively improve the student's academic record (Table I), reduce IAD (Table II), and improve psychological condition (Table III), as well as grades. The psychological quality improvement and enhancement of the self-control to use the Internet help students change their sedentary lifestyle by redirecting their time and energy to participate in extra-curricular activities actively, which leads to the formation of sports and exercise habits. This is more helpful to the students who spend a lot of time online. For example, an experiment conducted on junior high school students showed that physical exercise can play a positive role in junior high school students' learning self-control strategies, and the cumulative time with the best effect accounts for about $60 \%$ of the total physical education class time (Liu, 2017). Hu and Zhang (2016) found that the short-term exercise intervention could make mild Internet addicted students have better intervention effect as long as the interest of students was identified for rapid intervention. The number of students with severe IAD did not decrease significantly until 4 to 9 months after the exercise intervention, indicating that they need longer exercise intervention. However, as long as the students with severe IAD form the interest and habit of exercise, the effect of exercise intervention on the withdrawal of IAD is indisputable.
It can be seen that emergent learning theory is a new learning paradigm emerged with the development of the Internet, which has the "dual role" of maintaining and improving students' health and improving their academic performances.

\section{CONCLUSION}

Firstly, emergent learning theory is a new paradigm of learning theory that is not only conducive to improving students' academic performance, but also conducive to mental and physical health.

Secondly, emergent learning theory plays a unique role in developing students' cognitive abilities i.e. memory, attention, imagination, insight and logical thinking.

Thirdly, emergent learning theory is an effective means to correct IAD, sitting addiction and other bad behaviors and lifestyle, which is conducive to cultivating students' good learning habits and physical exercise habits, so as to form a positive psychological state.

Fourthly, the application of emergent learning theory can fully stimulate the positive effects of the Internet and curb the negative effects, so as to effectively overcome the "twoedged sword effect of the Internet", and ultimately enable human beings to achieve the goal of "control the Internet but not be slaved".

Fifthly, the mechanism of emergent learning theory: Integrate three kinds of behaviors of learning to think, exercising, reducing the use of the Internet. Make the brain and body work together, promote each other so that one can enhance his physique, consolidate learning content and become free from indulging in the Internet at the same time.

\section{ACKNOWLEDGMENT}

This work was supported by The Humanities and Social Sciences Planning Fund Project of Ministry of Education in China (09YJAZH018), Key Project of School Sports Scientific Research in Shanghai (HJTY-2016-C02) and Humanities and Social Sciences Interdisciplinary Project of Tongji University (1430219042).

\section{REFERENCES}

[1] Cedernaes, J., Rångtell, F. H., Axelsson, E. K., et al. Short Sleep Makes Declarative Memories Vulnerable to Stress in Humans. Sleep, 2015, 38 (12): 1861-1868.

[2] Chen, Shuhui, Weng, Lizhen, Su, Yiren, et al. Research on Chinese Internet addiction scale and its psychometric characteristics, Chinese Journal of Psychology, 2003, 45 (3): 279-294.

[3] Chen, Weidong, Yang, Xue. Empathic teaching design based on the advantage effect of pictures, Modern Education Technology, 2014, 24 (11): 23-29.

[4] Ding, Xuanhong, Zhang, Gongting, Li, Gang, et al. An investigation into the physical and mental health of young "phub-heads", Journal of Youth, 2015 (2): 36-40.

[5] Elaine, M. Murtagh, Linda, Nichols, Mohammed A. Mohammed, et al. The effect of walking on risk factors for cardiovascular disease: An updated systematic review and meta-analysis of randomised control trials. Preventive Medicine, 2015, 72: 34-43. 
[6] Hermann, Ebbinghaus. Memory: A contribution to experimental psychology. Germany: Hermann Ebbinghaus, 1885: 1-3.

[7] $\mathrm{Hu}$, Gengdan. Prevention and control measures of the adolescent constitution deterioration from the perspective of health management. London: Francis Academic Press, 2018.

[8] Hu, Gengdan, Qian, Longchao, Lin, Xiaobing. A preliminary study on the table tennis exercise treatment on Internet addiction disorder for juveniles. Dalian: Dalian University of Technology Press, 2013.

[9] $\mathrm{Hu}$, Gengdan, Xu, Quancheng. Internet Addiction Psycology. Beijing: Beijing Normal University Press, 2018.

[10] Hu, Gengdan, Zhang, Jun. Research on the role and mechanism of sports in correcting adolescent Internet addiction from the perspective of human instinct, China Sports Science and Technology, 2016, 52 (1): 68-77.

[11] Li, Dongping, Li, Xian, Wang, Yanhui, et al. School connectedness and problematic Internet use in adolescents: A moderated mediation model of deviant peer affiliation and self-control, Journal of Abnormal Child Psychology, 2013, 41: 1231-1242.

[12] Li, Wei, Yuan, Zhiguo. Playing mobile phones can also kill people. Xinhua Daily Telegraph, 2013-10-17 (005).

[13] Liu, Siliang. Experimental study on the influence of physical exercise on the learning self-control and academic performance of junior high school students. Yangzhou University, 2017.

[14] Ma, Siyuan. Research on physical deterioration of primary and secondary school students in China and its social causes. Beijing Sports University, 2012.

[15] Qian, Jianming. Psychological problems of excellent middle school students and education countermeasures. Nanjing Normal University, 2004.

[16] Scudder, M. R., Federmeier, K. D., Raine, L. B., et al. The Association between aerobic fitness and language processing in children: Implications for academic achievement. The Brain and Cognition, 2014, 87: 140-152.

[17] Shen, Kecai, Wu, Aijun. Leveraging the solid ice of teenagers' physical health with college entrance examination sports, Chinese School Sports, 2012 (7): 12-13.

[18] Sun, Jiangping. Juvenile and child health: Education textbook for national adult higher medical education. Beijing: People's Health Publishing House, 2000.

[19] Tao, Ran, Li, Bonghe. Overview of treatment units for Internet addiction, Chinese Journal of Epidemiology, 2005, 26 (8): 558.

[20] Wang, Lihao, Tong, Jiehui. Research on the relationship between college students' Internet addiction and social support, communication anxiety and self-harmony, China Journal of Health Psychology, 2003, 11(2): 94-96.

[21] Wang, Zhengyu. Symptom self-rating scale (SCL-90), Shanghai Psychiatric Medicine, 1984, 2 (2): 68.

[22] Wen, Xue, Hu, Zhongping. "Education load reduction" from the perspective of game theory, Chinese journal of education, 2007 (1): 22-24.

[23] Yang, Bing. Exploration on transforming "mechanical memory" into "understanding memory", Education Theory and Practice, 2008, 28 (S1): 89-91.

[24] Zhang, Chao-an. Rational thinking on comprehensive reform of youth sports, Journal of Beijing Sports University, 2014, 34 (8): 1-5+11.

[25] Zhang, Liwei, Mao, Zhixiong. The relationship between physical exercise and mental health (review), Journal of Guangzhou Physical Education Institute, 1995, 15 (4): 42-47. 